\title{
IN THE SWARM
}

\section{Dear Reader,}

Few other subjects are currently being as fiercely debated as highly automated or even fully autonomous driving. Partially automated assistance systems with dynamic lateral and longitudinal guidance such as ACC and lane keeping systems, which allow driving to become partially automated, will soon be part of the standard repertoire in automotive engineering. The systems that are already available enjoy a high level of acceptance by customers. However, increasing urbanisation and the future growth of conurbations worldwide will have far-reaching consequences for individual mobility. Engineers and consumers must accept the fact that individual transport in its current form, at least in urban environments, is a thing of the past. Cooperative mobility concepts will revolutionise driving.

That also makes sense, because increasing overcrowding of the main traffic routes will result in longer journey times, a higher risk of accidents and greater pollution of the atmosphere, and is therefore not economically sustainable. In the meantime, research and testing in the field of car-to-x systems is so far advanced that one can already speak of series-production readiness for the technologies required. Telematics, intelligent assistance systems and mobile communication systems are already capable of forming a comprehensive, dynamic traffic control system in real time, with ranges of up to $1000 \mathrm{~m}$ and driving speeds of up to $200 \mathrm{~km} / \mathrm{h}$.

From a technical point of view, these systems fulfil almost all of the requirements for communicating information on traffic jams, hazardous situations or accidents quickly and safely from vehicle to vehicle and initiating the necessary responses without intervention by the driver. In the first step, it is likely that only the vehicles will communicate with each other and inform each other (and their drivers) about situations. Nevertheless, it does not take a crystal ball to prophesy that it is then only a small step to autonomous actions being performed by the vehicle itself and its assistance systems. The emergency braking assistants that are currently offered by various manufacturers give a clear indication of how systems can take over - in a positive sense - if drivers are unable to control their vehicle quickly enough.

The technology is now available, but one serious problem still remains: according to current legislation, a fully autonomous vehicle would not be eligible for homologation. It is therefore up to politicians to prepare the ground to enable cooperative mobility based on a viable traffic control system to be quickly and sustainably implemented. Fluid, safe and fast control of complex traffic flows requires the intelligent control of moving masses and anyone who has observed a huge shoal of fish moving in perfect harmony will know what that looks like.

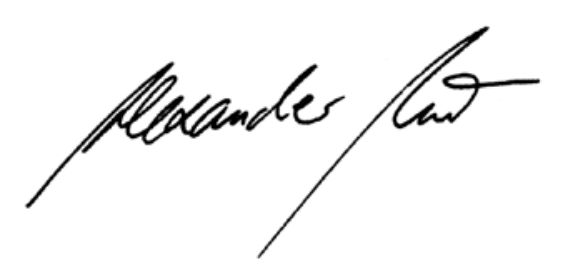

DR. ALEXANDER HEINTZEL, Editor in Chief Wiesbaden, 24 October 2013

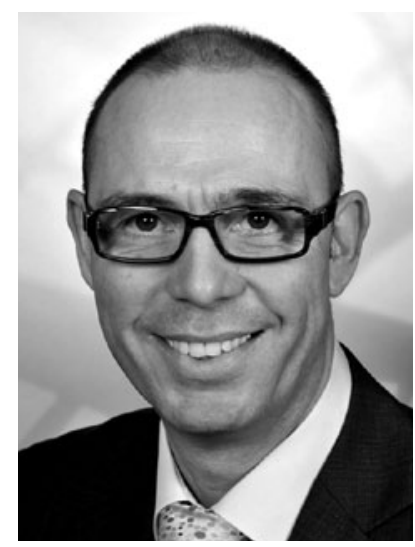

